\title{
Network Selection with Joint Vertical and Horizontal Handoff in Heterogeneous WLAN and Mobile WiMax Systems
}

\author{
Li-Chun Wang*, Anderson Chen and Hung-Hsi Chen
}

\begin{abstract}
As the IEEE 802.11 wireless local area networks (WLANs) have become very popular and the emerging IEEE 802.16e wireless metropolitan area networks (WMANs) also attract a great deal of attentions, in the future we can expect the appearance of the hybrid IEEE 802.11 WLANs and IEEE 802.16e WMANs systems. Clearly, seamless handoffs in this hybrid network will become an increasingly important issue. However, the system which has shortest handoff latency may not always have the highest throughput. In this paper, we develop a dynamic network selection scheme to determine the connecting system in a new "WLAN to hybrid WLAN/WiMax" handoff scenario, in which we consider both the vertical and horizontal handoffs. The proposed scheme requests the station to wait an additional network selection time " $t_{w}$ " before the selection to maximize the amount of delivered bits during a dwelling time. We also formulate an optimization problem to find the optimal network selection time $t_{w}^{*}$ and show that the proposed scheme can improve the delivered information bits during handoff.
\end{abstract}

\section{INTRODUCTION}

As the rapid progress of communication techniques in recent years, various wireless networks have been widely deployed. One can imagine that the future wireless access network will consist of wireless networks with various transmission rates and coverage ranges. For example, the IEEE 802.11 wireless local area network (WLAN) can provide high data rate per user and low deployment cost [1], [2]. On the other hand, the IEEE 802.16e wireless metropolitan area network (WMAN) can offer wide coverage range per cell and guarantee the quality of service requirement [3]. The hybrid WLAN and WMAN system can take advantages of both systems to provide high quality services. Therefore, the handoff in the hybrid IEEE 802.11 WLAN and IEEE 802.16e WMAN system becomes an important issue for the future wireless access networks.

In the literature, many studies have considered two conventional handoff scenarios, i.e. "WMAN to WLAN" and "WLAN to WMAN", as shown in Fig. 1(a). The objective in the first handoff scenario relates to the so called "always best connect" (ABC) [4]. Because a mobile user can concurrently connect to both the WLAN and WMAN, it requires to choose which system is the best for its service requirement. Some papers proposed cost function based vertical handoff decision

This work was jointly supported by the National Science Council, the Program of Promoting University Excellence of Ministry of Education, Taiwan and Zyxel Inc. under the contracts NSC94-2213-E-009-060, EX-91-E-FA064-4 and 94C033.

The authors are with the Department of Communication Engineering, National Chiao-Tung University, Taiwan, R.O.C. (e-mail: lichun@cc.nctu.edu.tw; mingbing.cm87g@nctu.edu.tw)

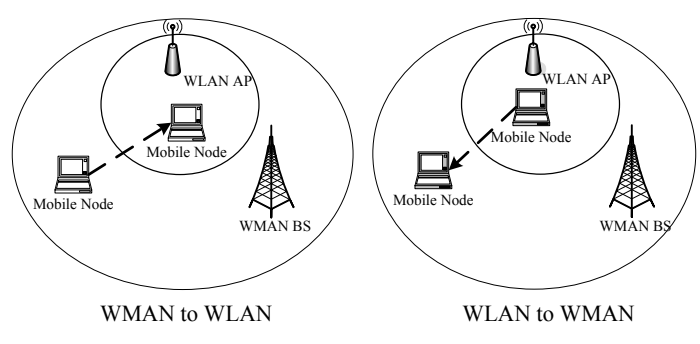

(a)

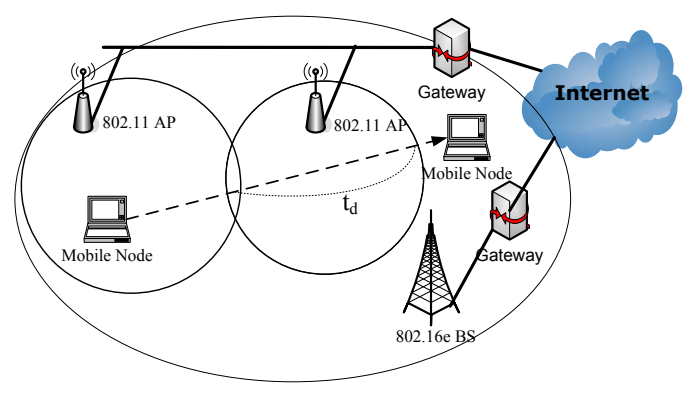

(b)

Fig. 1. (a) Two traditional vertical handoff scenarios, i.e. WMAN to WLAN and WLAN to WMAN (b) New WLAN to WLAN/WMAN handoff scenario.

algorithms to achieve the "ABC" objective in this handoff scenario [5], [6]. However, to accurately obtain performance metrics anytime, such as available data rate, access latency and etc., is not easy for a mobile user in the first "WLAN to WMAN" handoff scenario with a mobile user in the overlapped region covered by both WLAN and WMAN. On the other hand, in the second "WLAN to WMAN" handoff scenario when a WLAN user enters a region covered only by WMAN, the "seamless" handoff issue is crucial because the mobile user needs fast transiting connections to the new WMAN in order to minimize the link disconnected time [7][9]. In [9], [10], the authors proposed the vertical handoff mechanisms which adopt fast Fourier transform for handoff detection and the dwelling timer concept to postpone the 
TABLE I

LINK-LAYER HANDOFF TIME FOR VARIOUS WLAN CARDS [11]

\begin{tabular}{|c|c|c|c|c|}
\hline & D-Link 520 & Spectrum24 & ZoomAir & Orinoco \\
\hline \hline Search & $288 \mathrm{~ms}$ & $98 \mathrm{~ms}$ & $263 \mathrm{~ms}$ & $87 \mathrm{~ms}$ \\
\hline Execution & $2 \mathrm{~ms}$ & $3 \mathrm{~ms}$ & $2 \mathrm{~ms}$ & $1 \mathrm{~ms}$ \\
\hline Total & $290 \mathrm{~ms}$ & $101 \mathrm{~ms}$ & $265 \mathrm{~ms}$ & $88 \mathrm{~ms}$ \\
\hline
\end{tabular}

handoff decision in order to avoid unnecessary handoff and accurately make the decision.

However, since the WLAN has been widely deployed and the WiMax with wide coverage is dramatically developing in these years, one can imagine that the WiMax system will overlay on the existing WLAN in the near future. Thus, a new "WLAN to WLAN/WiMax" handoff scenario occurs as shown in Fig. 1(b). This new handoff scenario in the hybrid WLAN and WiMax system jointly considers the seamless issue in the horizontal and vertical handoff, i.e. "WLAN to WLAN" and "WLAN to WiMax", respectively. Furthermore, the mobile station is requireed to select an appropriate network between WLAN and WiMax during handoff. Therefore, in addition to the seamless issue, the $\mathrm{ABC}$ is also another important problem to deal with at the same time in this new handoff scenario.

In this paper, we develop a network selection scheme in the new "WLAN to WLAN/WiMax" handoff scenario to determine which system has the most delivered information bits during the dwelling time $t_{d}$. The proposed scheme requests the handoff station awaiting for a short network selection time $t_{w}$ before selecting the networks. The rests of this paper are organized as follows. Section II gives a background on the channel search process in WLAN and awakening process of sleep mode in WiMax. Section III introduces the system overview on the proposed network selection scheme. Section IV formulates the problem and analyzes the delivered information bits during the dwelling time. Section V shows the numerical results, and the concluding remarks are given in section VI.

\section{BACKGROUND}

In this section, we first introduce channel search and awakening process, which are the most important steps during the WLAN and WMAN handoff procedures.

\section{A. The Channel Search Process in the IEEE 802.11}

The handoff procedures in the IEEE 802.11 WLAN can be divided into two phases: channel search and execution phases [1]. In the phase of channel search, the handoff station broadcasts probe request frames at each channel to acquire the AP information. Then, the AP replies a probe response frame to inform the handoff station upon receiving the probe request frame. After that, the handoff station selects one appropriate AP to establish a new connection in the execution phase. From the experiments in some research studies, the latency in the channel search phase dominates the total link-layer handoff time as shown in Table I [11], [12]. Therefore, in this paper, we only consider the impact of channel search process on

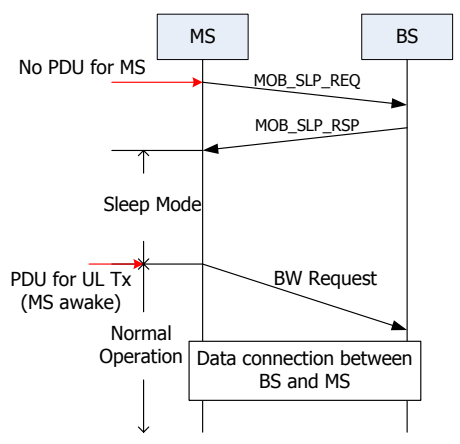

Fig. 2. Transition signaling of station initiation in IEEE 802.16e sleep mode.

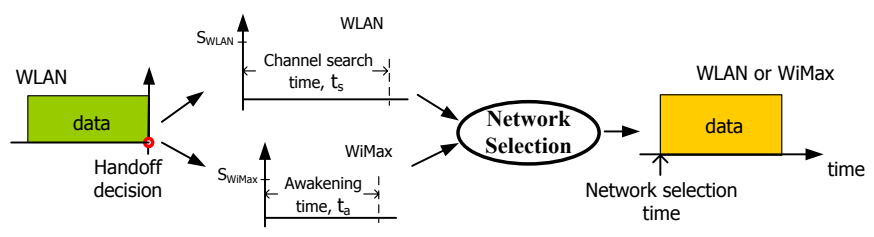

Fig. 3. System model for jointly vertical and horizontal handoff (WLAN to WLAN/WiMax).

the WLAN horizontal handoff in the considered "WLAN to WLAN/WiMax" scenario.

\section{B. The Awakening Process in IEEE 802.16e}

In the hybrid WLAN and WMAN system, the coverage area of several WLANs can be overlapped by the coverage of a WMAN, as shown in Fig. 1(b). Consider a mobile station with dual interfaces consisting of IEEE 802.11 and IEEE 802.16e. It can be assumed that this mobile stations stays in the WMAN coverage so that the network re-entry process is unnecessary. When the station switches the connection to WLAN, the WiMax interface can change to the sleep mode as shown in Fig. 2. To restore the data connection, the WiMax interface follows the random access procedures and merely sends the bandwidth request frame [3], [13]. Therefore, in this paper, we consider the only influence of the awakening process in the "WLAN to WiMax" handoff scenario.

\section{SYSTEM OVERVIEW}

Next, we describe the considered system model in this paper and the proposed network selection scheme for the new "WLAN to WLAN/WiMax" handoff scenario.

\section{A. System Model}

Figure 1(b) illustrates a new "WLAN to WLAN/WiMax" handoff scenario considered in this paper. Let a dual-interface mobile station switch to WLAN for transmitting data, while preserving a connection in the IEEE 802.16e sleep mode [3], [14]. As the station moves and the signal strength received from the serving AP is below a predefined threshold, the channel search and awakening processes in WLAN and 


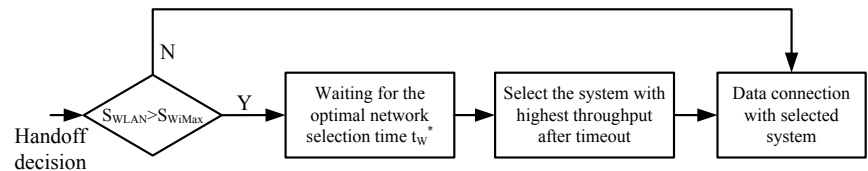

Fig. 4. Proposed network selection scheme in the WLAN to WLAN/WiMax handoff scenario.

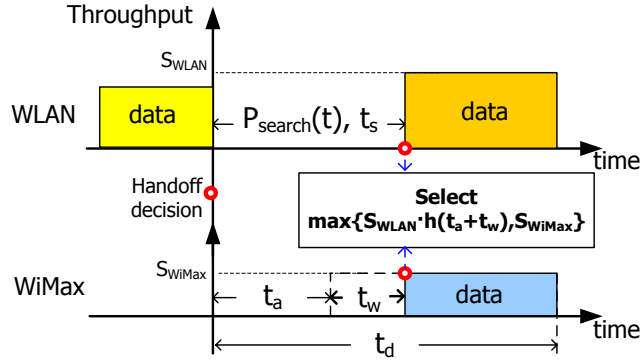

Fig. 5. Timing diagram of network selection between WLAN and WiMax.

WiMax will be respectively executed, as shown in Fig. 3. At last, the station performs the network selection procedure to determine the appropriate system that has the maximum delivered information bits during the dwelling time $t_{d}$.

\section{B. Proposed Network Selection Scheme}

The proposed network selection scheme between WLAN and WiMax is depicted in Fig. 4. Assume that the WiMax system successfully establishes the connection first. Then, if the per user throughput in WiMax is higher than that in WLAN, the station creates the data connection with WiMax immediately. Otherwise, the mobile station waits the optimal network selection time $t_{w}^{*}$ before selecting the system with the highest per user throughput. At last, the station creates the data connection with selected system.

\section{PERFORMANCE ANALYSis}

\section{A. Problem Formulation}

Because both WLAN and WMAN use distinct access techniques, the channel search time $t_{s}$ in WLAN differs from the awakening time $t_{a}$ in WiMax. In addition, the available per user throughput in WLAN and WiMax, respectively denoted

TABLE II

A EXAMPLE FOR PERFORMANCE IN WLAN TO WLAN/WIMAX

\begin{tabular}{|c|c|c|c|}
\hline & $\begin{array}{c}\text { number of stations } \\
\text { in target network }\end{array}$ & $\begin{array}{c}\text { MAC throughput } \\
\text { per user }\end{array}$ & $\begin{array}{c}\text { handoff } \\
\text { latency }\end{array}$ \\
\hline $\begin{array}{c}\text { WLAN to WLAN } \\
\text { (horizontal) }\end{array}$ & 10 (user density: & $4.35 \mathrm{Mbps}$ & $30 \mathrm{~ms}$ \\
$\left(t_{s}\right)$
\end{tabular}

Note: The coverages of WLAN and WiMax are 100 and 1400 meters, respectively. as $S_{\text {WLAN }}$ and $S_{\text {wiMax }}$, are also different due to different coverage. We can expect that a handoff station can deliver more information bits if it can postpone the network selection for a short duration after one of the interfaces successfully establishing a new connect. From Table II, we find that WiMax has short awakening latency, i.e. $t_{a}=10 \mathrm{~ms}$, but has lower available per user throughput than that in WLAN. Therefore, the problem is how long the handoff station has to wait before selecting the networks so that it can maximize the delivered bits during the dwelling time.

Figure 5 illustrates the timing diagram of the network selection in the hybrid WLAN and WiMax system. After waiting a network selection time $t_{w}$, the handoff station selects the system $i$ with the highest available throughput according to

$i=\left\{\begin{array}{ll}\text { WLAN, } & \text { if } S_{\mathrm{wLAN}}\left(n_{11}\right) \cdot h\left(t_{a}+t_{w}\right) \geq S_{\text {WiMax }}\left(n_{16}\right) \\ \text { WiMax, } & \text { otherwise }\end{array}\right.$,

where $n_{11}$ and $n_{16}$ are the number of stations in the target WLAN and WMAN, respectively. The function $h\left(t_{a}+t_{w}\right)$ indicates the event that the channel search process in WLAN is successful after a duration of $t_{a}+t_{w}$, i.e.

$h\left(t_{a}+t_{w}\right)= \begin{cases}1, & \text { if WLAN channel search succeed } \\ & \text { after } t_{a}+t_{w} \\ 0, & \text { otherwise }\end{cases}$

However, the dwelling time $\left(t_{d}\right)$ in the target network is limited, and the station may not always succeed in the channel search process of WLAN. Thus, the previous problem is equivalent to find the optimal network selection time " $t_{w}^{*}$ " under the delay constraint $d_{i}$ such that the delivered bits during the dwelling time $t_{d}$ can be maximized, i.e.,

$$
\begin{aligned}
t_{w}^{*}=\arg \max _{0 \leqslant t_{w} \leqslant d_{i}-t_{a}}\left\{\left[P_{\text {search }}\left(t_{w}+t_{a}\right) \cdot S_{\mathrm{WLAN}}\left(n_{11}\right)\right.\right. \\
\left.\quad+\left(1-P_{\text {search }}\left(t_{w}+t_{a}\right)\right) \cdot S_{\mathrm{WiMax}}\left(n_{16}\right)\right] \\
\left.\cdot\left(t_{d}-t_{w}-t_{a}\right)\right\}
\end{aligned}
$$

where $P_{\text {search }}\left(t_{a}+t_{w}\right)$ is the success probability during the channel search time $t_{a}+t_{w}$ in WLAN.

\section{B. Delivered Information Bits, $S\left(t_{w}\right)$}

Next, we analyze the impact of network selection time $t_{w}$ on the delivered information bits during the dwelling time $t_{d}$ in the new "WLAN to WLAN/WiMax" handoff scenario. According to [15], given a period of time, $t$, we can obtain a successful search probability value, $P_{\text {search }}(t)$. Thus, the total delivered information bits " $S\left(t_{w}\right)$ " in both WLAN and WiMax systems during the dwelling time $t_{d}$ is given by

$$
\begin{aligned}
S\left(t_{w}\right)= & g\left(t_{a}\right) \cdot\left(t_{d}-t_{a}-t_{w}\right) \cdot\left[P_{\text {search }}\left(t_{a}+t_{w}\right)\right. \\
& \left.\cdot S_{\mathrm{WLAN}}+\left(1-P_{\text {search }}\left(t_{a}+t_{w}\right)\right) \cdot S_{\mathrm{WiMax}}\right] \\
& +\left(1-g\left(t_{a}\right)\right) \cdot\left(t_{d}-t_{a}\right) \cdot S_{\mathrm{WiMax}}
\end{aligned}
$$

where

$$
g\left(t_{a}\right)= \begin{cases}1, & \text { if } S_{\mathrm{WLAN}}\left(n_{11}\right) \geq S_{\mathrm{WiMax}}\left(n_{16}\right) \text { after } t_{a} \\ 0, & \text { otherwise }\end{cases}
$$


TABLE III

SYSTEM PARAMETERS

\begin{tabular}{|c|c|}
\hline MAC throughput per user in WLAN [16] & $\begin{array}{c}4.25 \mathrm{Mbps} \text { (10 users) } \\
1.22 \mathrm{Mbps} \text { (30 users) }\end{array}$ \\
\hline MAC throughput per user in WiMax [17] & $1.11 \mathrm{Mbps}(50 \mathrm{users})$ \\
\hline awakening time in WiMax & $25 \mathrm{~ms}$ \\
\hline number of channels in WLAN & 3 \\
\hline MS moving speed & $5 \mathrm{~km} / \mathrm{hr}$ \\
\hline WLAN coverage & $100 \mathrm{~m}$ \\
\hline dwelling time in target WLAN $\left(t_{d}\right)$ & $72 \mathrm{sec}$ \\
\hline
\end{tabular}

\section{Numerical RESUlts}

In this section, we examine the total delivered information bits during the dwelling time $t_{d}$ by means of various network selection schemes. We also list the maximum delivered information bits after awaiting the optimal network selection time " $t_{w}^{*}$ " with different number of stations. The considered network topology is shown in Fig. 1(b), where 50 stations are located in WMAN. A handoff station moves from one WLAN to another WLAN at the speed of $5 \mathrm{~km} / \mathrm{hr}$, and it looks for the AP among the three channels in WLAN. The coverage of a WLAN is 100 meters, and the WMAN covers both the two WLANs. Other related system parameters are shown in Table III.

\section{A. Delivered bits in the proposed and conventional schemes}

Figure 6 shows the total delivered information bits during the dwelling time $t_{d}$ versus different network selection time $t_{w}$ with 10 and 30 stations in the target WLAN, respectively. The considered two network selection schemes are: (1) dynamic network selection among WLAN and WiMax; (2) persistent WLAN selection, i.e., the handoff station insists on connecting to the target WLAN. In addition, the figures also show the amount of delivered information bits if a station stays in WLAN and WiMax during the dwelling time $t_{d}$, respectively. As shown in Fig. 6(a), the dynamic selection scheme only improves $9 \%$ for the case of 10 stations in the target WLAN. Due to fewer contentions between the handoff station and others in the target WLAN, the handoff station can easily find an available AP and thus the improvement decreases. However, in the case of 30 stations, the proposed scheme improves almost by $100 \%$ compared to the "persistent WLAN selection" scheme, as shown in Fig. 6(b). In this situation, the target WLAN has low available throughput and the channel search time is long due to the increase of the contentions. Therefore, the dynamic selection scheme in the crowded WLAN outperforms than that in sparse WLAN.

\section{B. Optimal network selection time}

Figure 7 shows the impacts of the network selection time $t_{w}$ on the delivered information bits. As shown in the figure, an optimal network selection time $t_{w}^{*}$ exists to enable the handoff station delivering maximum information bits during the dwelling time, $t_{d}$. As the network selection time $t_{w}$ prolongs, the amount of delivered information bits increases due to the improvement of success probability in the channel

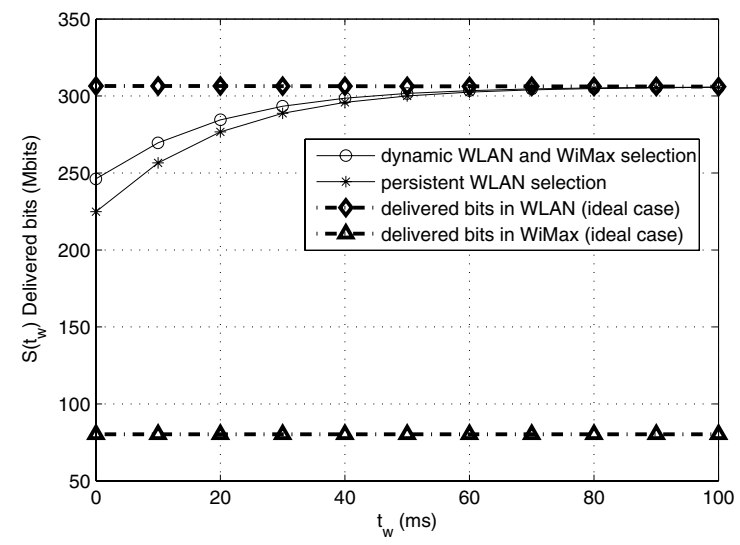

(a)

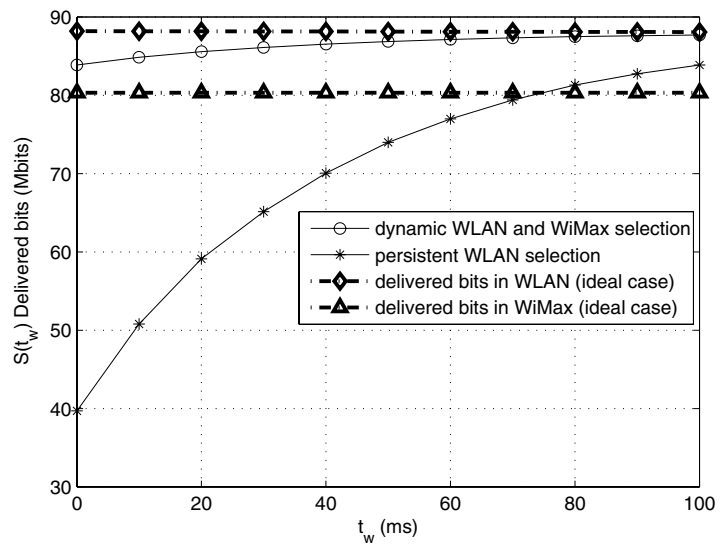

(b)

Fig. 6. The amount of delivered bits during $t_{d}$ in proposed and conventional schemes with (a) 10 and (b) 30 stations in the target WLAN, respectively.

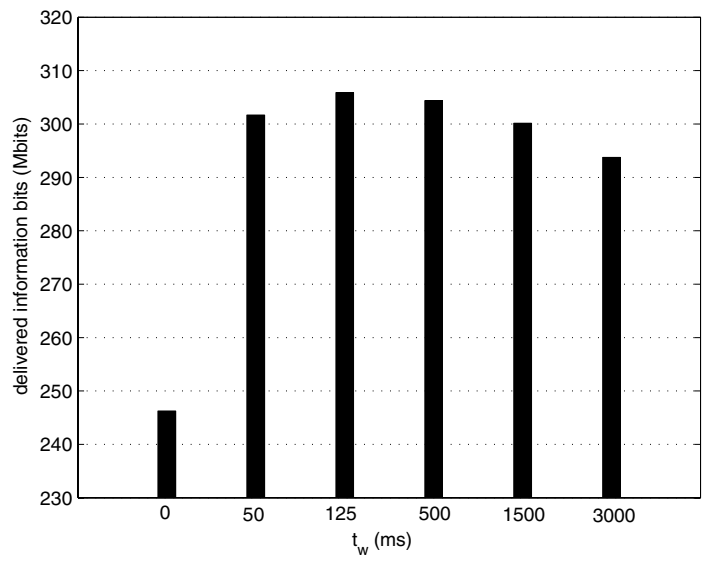

Fig. 7. The amount of delivered bits versus the various network selection time $t_{w}$ with 10 stations in the target WLAN. 
TABLE IV

OPTIMAL NETWORK SELECTION TIME $t_{w}^{*}$

\begin{tabular}{|c|c|c|}
\hline $\begin{array}{c}\text { number of } \\
\text { stations }\left(n_{11}\right)\end{array}$ & $\begin{array}{c}\text { optimal network selection } \\
\text { time } t_{w}^{*}(\mathrm{~ms})\end{array}$ & $\begin{array}{c}\text { maximum delivered } \\
\text { bits (Mbits) }\end{array}$ \\
\hline 5 & 80 & 659 \\
\hline 10 & 125 & 306 \\
\hline 15 & 170 & 194 \\
\hline 30 & 185 & 88 \\
\hline
\end{tabular}

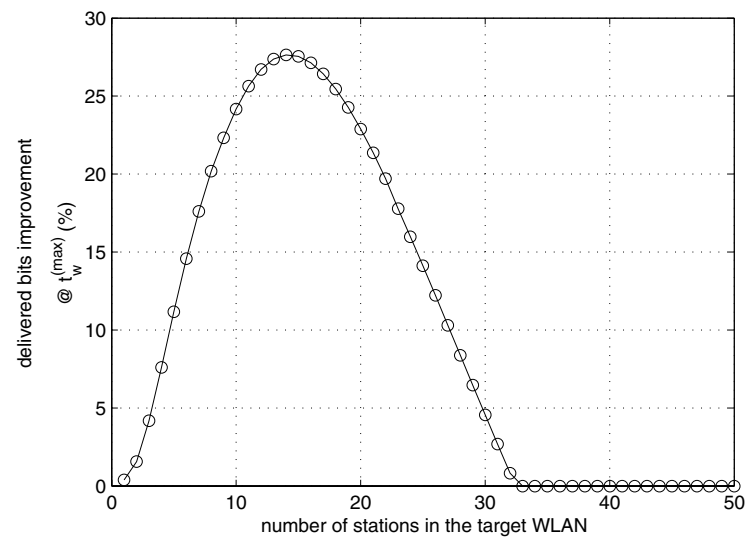

Fig. 8. Improvement of delivered bits with optimal network selection time $t_{w}^{*}$ versus number of stations in the target WLAN.

search process. However, when the duration of $t_{w}$ is too large, the amount of delivered bits saturates and even starts decreasing because of the long wasted time in channel search. Thus, after awaiting only an optimal network selection time $t_{w}^{*}$, the handoff station can maximize the delivered information bits during the dwelling time, $t_{d}$. Table IV lists the values of the optimal network selection time $t_{w}^{*}$ and the corresponding delivered bits with various numbers of stations in WLAN.

\section{Delivered Bits Improvement}

Figure 8 shows the improvement of the delivered information bits at the optimal network selection time $t_{w}^{*}$ with various numbers of stations in WLAN compared to that at $t_{w}=0$. As shown in the figure, when $5 \sim 25$ stations are located in the target WLAN, the handoff station waiting for the optimal network selection time $t_{w}^{*}$ can deliver at least more $10 \%$ information bits. However, in the sparse WLAN, e.g. 5 stations in the target WLAN, the improvement is small due to the high successful channel search probability. On the other hand, as the network is more crowded, e.g., more than 30 stations, the handoff station does not need to search the WLAN because the available throughput in WLAN is smaller than that in WiMax.

\section{CONCLUSIONS}

In this paper, we identified a new "WLAN to WLAN/WiMax" handoff scenario. A dynamic WLAN/WiMax network selection scheme was proposed to maximize the delivered information bits during the dwelling time $t_{d}$ in a new "WLAN to WLAN/WiMax" handoff scenario. The proposed scheme requests the handoff station awaiting a short network selection time $t_{w}$ to maximize the delivered bits during the dwelling time $t_{d}$ before selecting the target networks. Our numerical results showed that the number of delivered bit are improved by $10 \%$ compared to the tradition scheme.

\section{REFERENCES}

[1] IEEE 802.11, Part 11: Wireless LAN Medium Access Control (MAC) and Physical Layer (PHY) Specifications, Standard, IEEE, Aug. 1997.

[2] IEEE 802.11g, Part 11: Wireless LAN Medium Access Control (MAC) and Physical Layer (PHY) specifications: Further Higher Data Rate Extension in the $2.4 \mathrm{GHz}$ Band, supplement to IEEE 802.11 Standard, June 2003.

[3] IEEE 802.16e, Part 16: Air Interface for Fixed and Mobile Broadband Wireless Access Systems Amendment 2:Physical and Medium Access Control Layers for Combined Fixed and Mobile Operation in Licensed Bands, Dec. 2005.

[4] E. Gustafsson and A. Jonsson, "Always best connect," IEEE Wireless Communications, vol. 10, pp. 49 - 55, Feb. 2003.

[5] F. Zhu and J. McNair, "Optimizations for vertical handoff decision algorithm," IEEE Wireless Communications and Networking Conference, vol. 2, pp. $867-872$, March. 2004.

[6] M. J. and F. Zhu, "Vertical handoffs in fourth-generation multinetwork environments," IEEE Wireless Communications, vol. 11, pp. 8 - 15, March. 2004

[7] Z. A.H. and B. Liang, "Performance evaluation framework for vertical handoff algorithms in heterogeneous networks," IEEE International Conference on Communications, vol. 1, pp. 173 - 178, May 2005.

[8] A. R. Inayat R. and N. K., "A seamless handoff for dual-interfaced mobile devices in hybrid wireless access networks," Advanced Information Networking and Application, vol. 1, pp. 373 - 378, 2004.

[9] Q. Z. Chuanxiong Guo, Zihua Guo and W. Zhu, "A seamless and proactive end-to-end mobility solution for roaming across heterogeneous wireless networks," IEEE Journal on Selected Areas in Communications, vol. 22, pp. 834 - 848, June 2004.

[10] M. J. Ylianttila M., Pande M. and M. P., "Optimization scheme for mobile users performing vertical handoffs between IEEE 802.11 and GPRS/EDGE networks," Proceedings of IEEE Global Telecommunications Conference, vol. 6, pp. 3439 - 3443, Nov. 2001.

[11] H. Velayos and G. Karlsson, "Techniques to reduce the IEEE 802.11b handoff time," IEEE International Conference on Communications, vol. 7, pp. 3844 - 3848, June 2004.

[12] A. Mishra, M. Shin, and W. Arbaugh, "An empirical analysis of the IEEE 802.11 MAC layer handoff process," ACM SIGCOMM Computer Communication, vol. 33, no. 2, pp. 93 - 102, April 2003.

[13] A. Chen and L.-C. Wang, "On the performance of re-entry process in OFDMA-based IEEE 802.16e WiMax," Wireless Personal Multimedia Communications, Sept. 2006, to be appeared.

[14] N.-H. Lee and S. Bahk, "MAC sleep mode control considering downlink traffic pattern and mobility," IEEE Vehicular Technology Conference, vol. 3, pp. 2076 - 2080, June 2005.

[15] L.-C. Wang, H.-H. Chen, A. Chen, and C.-J. Chang, "Optimal channel search time for handoff in the IEEE 802.11 WLANs," IEEE Vehicular Technology Conference, March. 2006.

[16] G. Bianchi, "Performance analysis of the IEEE 802.11 distributed coordination function," IEEE Journal on Selected Areas in Communications, vol. 18, no. 3, pp. 535-547, March 2000.

[17] C. Hoymann, "Analysis and performance evaluation of the OFDMbased metropolitan area network IEEE 802.16," Computer Networks, vol. 49, pp. 341 - 363, June 2005. 